\title{
A Geometric Maximum Principle for Surfaces of Preseribed Mean Curvature in Riemannian Manifolds ${ }^{0}$ )
}

\section{U. DIERKES}

Sei $M$ eine dreidimensionale Riemannsche Mannigfaltigkeit und / eine Fläche vorgeschriebener mittlerer Krümmung, die in einer Menge $j \cup S \subset M$ mit $S$ als Rand beschränkter mittlerer Krümmung $\mathfrak{S}$ liegt. Unter natürlichen Bedingungen wird bewiesen, daß $S$ völlig in $J$ liegt. Als unmittelbare Konsequenz dieses Resultats ergibt sich eine hinreichende Bedingung für die Existenz von Minimalflächen in einer Menge $J \subset \mathbb{R}^{3}$, deren Rand $S$ nicht $\mathfrak{W}$-konvex ist.

ПІусть $M$ трёхмерное римановое многообразие и пусть $f$ поверхность предписанной средней кривизны и лежащая в множестве $J \cup S \subset M$ с нраём $S$ ограниченной средней кривизны $\mathfrak{g}$. При естественных условиях доказывается, что $S$ лежит полностью в $J$. Как непосредственное сліпствие получается достаточное условие существования минимальных поверхностей в множестве $\bar{J} \subset \mathbb{R}^{3}$ край которых ие $\mathfrak{Q}$-выпукло.

Let $M$ be a three-dimensional Riemannian manifold and let $f$ be some surface of prescribed mean curvature which is restricted to lie in some set $J \cup S \subset M$ with boundary $S$ of bounded mean curvature $\mathfrak{Q}$. Assuming natural conditions, we prove that the image of / lies completely in $J$. An immediate consequence of this result is a sufficient condition for the existence of minimal surfaces in a set $J \subset \mathbb{R}^{3}$, the boundary $S$ of which is not $\mathfrak{G}$-convex.

\section{Introduction}

In this paper we shall derive an inclusion theorem for surfaces $f$ of prescribed mean curvature $H$ in a three-dimensional Riemannian manifold $M$. The decisive quantities which are involved in our result are the absolute values of both, the prescribed mean curvature $H$ and the mean curvature $\mathfrak{d}$ of the boundary $S$ of some including set $J$, the area of the surface $f$ and the distance from the boundary of $f$ to $S$. To be more precise, if $f: \Omega \rightarrow J \cup S \subset M$ is some conformally parametrized surface which is of prescribed mean curvature $H$ in the interior $J$; then there exists some constant $c=c(\Lambda, \tau, x, R)$ depending only on $\Lambda=\max \left\{|H|_{0},|\mathfrak{W}|_{0}\right\}$, the injectivity radius $\tau$, an upper bound for the sectional curvature $x$ and the distance $R=\operatorname{dist}_{M}(f(\partial \Omega), S)$ such that $f(\bar{\Omega}) \subset$ int $J$ provided the area of $f$ is smaller than $c$.

Thus the main emphasis of the theorem, which also distinguishes this result from the $\mathfrak{G}-\Lambda$ maximum principle by Hildebrandt [11], and Gulliver and Spruck [7], is the fact that the inward mean curvature $\mathfrak{W}$ of the boundary $S$ need not be greater than the absolute value of the prescribed mean curvature $H$. In particular we allow obstacles $S$ the (inward) mean curvature $\mathfrak{B}$ of which is negative. Exterior domains are therefore typical examples which fit.in our framework.

The analytic tool for the proof of our theorem is an estimate by GR ÜTER [5], who used a method from geometric measure theory to prove a pulled back version of the standard monotonicity formula.

0) AMS classification code: 49 F 10, 53 A 10, 35 A 15. 
In view of certain existence-regularity results of HLDEBRANDT and KAUL [13] and HILDEBRANDT [12] a direct consequence of the inclusion theorem is a new existence result for minimal surfaces in $\mathbb{R}^{3}$, which are restricted to lie in $J$ (Proposition 1). Again we are interested in cases where $S$ is not $\mathfrak{D}$-convex (i.e. $\mathfrak{W} \geqq 0$ is not satisfied): If $A_{\Gamma, J}$ is the infimum of area of surfaces spanned by the curve $\Gamma$ in $J$, then the condition is that $|\mathfrak{Q}|_{0}<\left\{-1 / 4 R^{2}+\pi / 2 A_{\Gamma . J}\right\}^{1 / 2}-1 / 2 R$. Two examples illustrate this result: Another application of Theorem 1 appears in Proposition 2, which sharpens a result of Böhme, HildebrandT and TAUSCH [1: Theorems 12, 13] concerning the existence of extremals for the integral $E(x)=\int x_{3}|\nabla x(u, v)|^{2} d \dot{u} d v$. Again a smallness condition on the quantity $A_{\Gamma . J}$ implies existence of an extremal for $E$.

A further application is treated in [3].

\section{Notations and results}

We shall adopt here the definition of $H$-surfaces in Riemannian manifolds given by Hildebrandt and KaUl [13], but, in short, repeat the basic concept. Let $M$ be a complete, connected and orientable Riemannian manifold of differentiability class three and $\Omega \subset \mathbb{R}^{2}$ be an open, connected and bounded set with Lipschitz boundary $\partial \Omega$ and with standard Euclidean metric, put $w=u+\mathrm{i} v$, and $u=u_{1}, v=u_{2}$. The Levi-Civita connection on $M$ will be denoted by $D$, furthermore $d: M \times M \rightarrow \mathbb{R}$ stands for the distance function on $M$ and $\|\cdot\|,\langle\cdot, \cdot\rangle$, denote the norm and the scalar product on $T_{p} M$, respectively. A function $f: \Omega \rightarrow M$ belongs to the class $H_{2}{ }^{1}(\Omega, M)$ if $f \in H_{2}{ }^{1}\left(\Omega, \mathbb{R}^{N}\right)$ and $f(\Omega-N) \subset M, N \subset \Omega$ denoting some null set (cp. [5: (2.1) Def.]). Here $M$ is thought to be isometrically embedded into some $\mathbb{R}^{N}$, and $H_{2}{ }^{1}\left(\Omega, \mathbb{R}^{N}\right)$ stands for the Sobolev. space of $L_{2}\left(\Omega, \mathbb{R}^{N}\right)$-functions the derivatives of which are again in $L_{2}$. The classes $H_{2}^{2}(\Omega, M)$ are defined similarly.

In the following let $M$ be three-diniensional and $\varphi: U \rightarrow \mathbb{R}^{3}$ denote some chart of an open set $U \subset M$. Then $x$ stands for the representation of $f$ corresponding to that chart. Furthermore, with respect to these coordinates, $g_{i k}$ and $\Gamma_{i j}^{k}$ denote the coefficients of the metric and the Christoffel symbols, respectively. Put $g:=\operatorname{det} g_{i k}$ and $g^{i k}:=\left(g_{i k}\right)^{-1}$. Consider now a function $\sigma \in C^{2}(M, \mathbb{R})$ and its level surface $S_{c}:=\{p \cdot \epsilon$ $M: \sigma(p)=c\}$, for $c \in \mathbb{R}$, as well as its "interior" $J_{c}:=\{p \in M: \sigma(p)<c\}$. Note that $S_{c}$ is $、$ regular, at $p$, provided $\operatorname{grad}_{p} \sigma \neq 0$. As usual the gradient vector field $\operatorname{grad}_{p} \sigma$ for $p \in M$ is given by $\left\langle\operatorname{grad}_{p} \sigma, V\right\rangle=V \sigma$ for any $V \in T_{p} M$. Also the Hessian tensor Hess $\sigma$, the Hessian bilinear form hess $\sigma$ and the Laplacian Lap $\sigma$ are defined by

$$
\begin{aligned}
& \operatorname{Hess}_{p} \sigma V=D_{V} \operatorname{grad}_{p} \sigma, \quad p \in M, V \in T_{p} M, \\
& \operatorname{hess}_{p} \sigma(V, W)=\left\langle\operatorname{Hess}_{p} \sigma V, W\right\rangle, V, W \in T_{p} M, \\
& \operatorname{Lap}_{p} \sigma=\operatorname{trace}\left(\operatorname{Hess}_{p} \sigma\right) .
\end{aligned}
$$

The mean curvature $\mathfrak{G}(p)$ of $S_{c}$ at $p$ with respect to the "interior normal" $-\operatorname{grad}_{p} \sigma /$ $\left\|\operatorname{grad}_{p} \sigma\right\|$ is defined by

$$
\mathfrak{\Phi}(p)=\frac{1}{2\left\|\operatorname{grad}_{p} \sigma\right\|}\left\{\operatorname{Lap} \sigma(p)-\frac{1}{\left\|\operatorname{grad}_{p} \sigma\right\|^{2}} \operatorname{hess}_{p} \sigma\left(\operatorname{grad}_{p} \sigma, \operatorname{grad}_{p} \sigma\right)\right\} .
$$

Consider a mapping $f \in H_{2,10 c}^{2}(\Omega, M) \cap H_{2}{ }^{1}(\Omega, M)$ and let $H=H(f)$ be a function of class $L_{\infty}(\Omega, \mathbb{R})$. Then $f$ is called an $H$-surface if it satisfies the equation

$$
D_{U_{a}} f_{*}\left(U_{\mathrm{a}}\right)=2 H(f(w)) f_{*}\left(U_{1}\right) \times f_{*}\left(U_{2}\right)
$$


and $\left\|f_{*}\left(U_{1}\right)\right\|=\left\|f_{*}\left(U_{2}\right)\right\|,\left\langle f_{*}\left(U_{1}\right), f_{*}\left(U_{2}\right)\right\rangle=0$ a.e. in $\Omega$. Here $U_{1}, U_{2}$ denote the basis fields with respect to $u_{1}, u_{2}$ and $f_{*}: T \Omega \rightarrow T M$ is the induced mapping of the tangent bundles. Moreover " $\times$ " denotes the cross product on $T_{p} M$. Let $w_{0} \in \Omega$ and $\Omega_{1} \subset \Omega$ be a neighbourhood of $w_{0}$ such that $f\left(\Omega_{1}\right)$ is contained in some coordinate neighbourhood $U \subset M$ with some chart $\varphi: U \rightarrow \mathbb{R}^{3}$. If $x=\varphi \circ f$ is the representation of $f$, then (1) implies

$$
\begin{aligned}
& \left.\Delta \dot{x}^{l}+\Gamma_{i j}^{l} x_{u \alpha}^{i} x_{u \alpha}^{j}=2 H(x(w)) g^{i k} \sqrt{g}\left(x_{u} \wedge x_{v}\right)_{k}{ }^{1}\right) \quad \text { a.e. on } \Omega_{1}(l=1,2 ; 3), \\
& g_{i j}(x) x_{u}^{i} x_{u}^{j}=g_{i j}(x) x_{v}^{i} x_{v}{ }^{j}, \quad g_{i j}(x) x_{u}^{i} x_{v}^{j}=0
\end{aligned}
$$

Here $\Delta=\partial^{2} / \partial u^{2}+\partial^{2} / \partial v^{2}$ denotes the (Euclidean) Laplacian. Note that $H$-surfaces are also weak $H$-surfaces in the sense of [5: cp. (3.5) Def.]: Moreover we use the abbreviations $D(f)=\int_{\Omega} g_{i j}(x) D_{a} x^{i} D_{a} x^{j} d u d v$. and $\Gamma=f(\partial \Omega)$ to denote the Dirichlet integral and the boundary, of $f$, respectively. Finally, put. $R=\operatorname{dist}\left(\Gamma, S_{c}\right)=\inf$ $\left\{d(\xi, \eta): \xi \in \Gamma, \eta \in S_{c}\right\} ; A(f)=$ area of $f, A=\max \left\{|H|_{0, \Omega},|\mathfrak{Q}|_{0, s_{c}}\right\}$, where

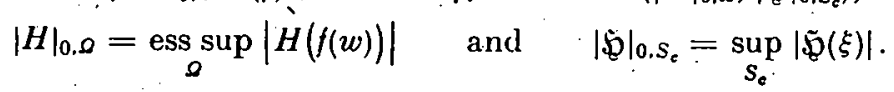

Let $\tau$ be the injectivity radius on $f(\Omega)$ and $x$ denote an upper bound for the sectional curvature on $f(\Omega)$ (for a precise definition and further properties concerning the injectivity radius and the sectional curvature we refer to GromolL, KLINGENBERg and Meyer [4].

Theoremi 1 : Let $\Omega, M, \sigma, J_{c}, S_{c}$ be defined as above. Assume that $f \neq$ const is some surface of class $H_{2}{ }^{1}(\Omega, M) \cap C^{0}(\bar{\Omega}, M) \cap H_{2,10 c}^{2}(\Omega, M)$ with the following properties:

$$
f(\Omega) \subset J_{c} \cup S_{c} \text {, }
$$

Then. $f(\bar{\Omega}) \subset J_{c}$ provided that either of the cases I or II holds:

(I) $x \leqq 0$ and $A(f)<\frac{\pi \varrho^{2}}{1+2 \Lambda \varrho+2^{-1}(2 \Lambda \varrho)^{2}}, \varrho:=\min \{R, \tau\}$.

$$
\text { (II) } x>0 \text { and } A(f)<\frac{\pi x^{-1}}{\frac{1}{\sin ^{2}(\varrho \sqrt{x})}+\frac{2 \Lambda \varrho}{\sin ^{2}(\varrho \sqrt{x})}+\frac{(2 \Lambda)^{2}}{x}}, \quad \varrho:=\min \left\{R, \tau, \frac{\pi}{2 \sqrt{x}}\right\} \text {. }
$$

Furthermore $f$ is of class $C^{k, \alpha}(\Omega, M)$ if $M$ belongs to $C^{k+1, \alpha}$ and $H$ is of class $C^{k-1, \alpha}(M, \mathbb{R})$,' $k \geqq 2$.

Remarks: 1 . Since $f$ is supposed to be continuous on $\bar{\Omega}$ we have $\tau>0$ and $x<\infty$. 2. If, in addition to the other hypotheses $M I$ is simply connected then case $I$ holds with $\varrho=R$ provided that $x \leqq 0$. In fact, this is a consequence of a theorem of Hadamard and Cartan, cf. [4: Section 7.2/Satz]. 8. In view of (iii) we find that $D(f) / 2=$ area of $f$. 4. The area of $f$ can be estimated by $L^{2}(\Gamma), L=$ length of $\Gamma=f(\partial \Omega)$, plus suitable error terms, cf. [14].

The following corollaries are simple consequences of the theorem.

Corollary 1: Suppose that $M$ is a simply connected, complete and orientable Riemannian manifold of class $C^{3}$ with non-positive sectional curvature and let $f \in C^{0}(\bar{\Omega}, M)$ $\cap H_{2,10 c}^{2}(\Omega, M) \cap H_{2}{ }^{1}(\Omega, M)$ satisfy conditions (i)-(iii) of Theorem 1 with $H \equiv 0$. Then

. 1) Here and in the sequel we agree to sum over repeated latin indices $i, j, k \ldots$ from 1 to 3 and over $\alpha, \beta$ from 1 to 2 : 
$f(\bar{\Omega}) \subset J_{c}$ is a minimal surface in $M$ provided that, in addition, $A(f)<\pi R^{2} /(1+2 \Lambda R$ $\left.+2^{-1}(2 \Lambda R)^{2}\right)$ where $\Lambda=|\mathfrak{W}|_{0, s_{c}}$ (Note that $M=\mathbb{R}^{3}$ is possible.)

Corollary 2: Let the assumption of Theorem 1 hold with $\varkappa \leqq 0$ and assume $\Lambda<\sqrt{\pi / A(f)}$. Then $f(\Omega) \subset J_{c}$ provided that $\sqrt{D} \frac{A \sqrt{D}+\sqrt{2 \pi-\Lambda^{2} D}}{2 \pi-2 \Lambda^{2} D}<\varrho, D=2 A(f)$.

Let $\Gamma \subset J \subset \mathbb{R}^{3}$ denote some closed Jordan arc, then the class $\subseteq(\Gamma, J)$ is defined by $\mathfrak{S}(\Gamma, J):=\left\{f \in H_{2}{ }^{1}\left(B, \mathbb{R}^{3}\right): f(\bar{B}) \subset \bar{J}\right.$.a.e., $\left.f\right|_{\partial B}: \partial B \rightarrow \Gamma$ is continuous and weakly monotonic $\}$, where $B=\left\{(u, v): u^{2}+v^{2}<1\right\}$. Put $A_{\Gamma, J}=2^{-1}$ inf $\{D(f): f \in \mathfrak{C}(\Gamma, J)\}$, then the existence-regularity results of $[12,13]$ together with Corollary 1 immediately lead to

Proposition 1: Let $\Gamma \subset \operatorname{int} J$ be a closed Jordan curve with $\subseteq(\Gamma, J) \neq \varnothing$ and suppose $S=\partial J$ is of class $C^{3}$, has bounded principal curvatures and a global parallel surface in $J$. If $A=|\mathfrak{W}|_{0, s}$ satisfies $A<\left\{-1 / 4 R^{2}+\pi / 2 \Lambda_{r . J}\right\}^{1 / 2}-1 / 2 R$, then there exists a minimal surface $h$ in $\dot{J}$, i.e. (i)-(iii) of Theorem 1 hold with $H \equiv 0, \Omega^{*}=\emptyset$.

Example. 1: Let $J$ be the torus of revolution which is generated by revolving the disk $\left(\xi_{1}-a\right)^{2}+\xi_{2}^{2}<r^{2}$ about the $\xi_{2}$-axis and assume $\Gamma$ permits $\mathfrak{C}(\Gamma, J) \neq \varnothing$. For $r<a<2 r$ the torus $S=\partial J$ has regions of negative inward mean curvature and thus the $\mathfrak{g}-\Lambda$ maximum principle by HILDEBRANDT [11] and GOLLIVER' and Spruck [6, 7] cannot be applied to solu-: tions of the variational problem $D(f)=\int|\nabla f(u, v)|^{2} d u d v \rightarrow$ minimum in $\mathfrak{E}(\Gamma, J)$. On the other hand the maximum absolute value of the mean curvature of $S$ is given by $A_{0}=2^{-1} \max$ $\{(a+2 r) / r(a+r),|a-2 r| / r(a-r)\}$. Proposition 1 gives the existence of a minimal surface spanned by $\Gamma$ in $J$ provided $A_{r, J}$ and $R$ satisfy $\Lambda_{0}<\left\{-1 / 4 R^{2}+\pi / 2 A_{\Gamma, J}\right\}^{1 / 2}-1 / 2 R$. To obtain a numerical example one may assume further that $\Gamma$ is contained in the torus of revolution that is generated by the disk $\left(\xi_{1}-a\right)^{2}+{\xi_{2}}^{2} \leqq(0.8 r)^{2}$ and that $r=2, a=3$. Then $R=2 / 5$ leads to the sufficient condition $A_{\Gamma, J} \leqq 0.41$.

Example 2: Let $J=\left\{\xi \in \mathbb{R}^{3}:|\xi| \geqq 1\right\}$ be the exterior of the unit ball. Then $\mathscr{G}=-1$, $A=1$ and for $R \geqq 1$ Proposition 1 gives the existence of a minimal surface spanned by $\Gamma$ in $J$ if $A_{\Gamma, J}<\pi / 5$. Note that the critical value for $A_{\Gamma, J}$ in this configuration is $3 \pi$, since the disk spanned by the circle $\left\{\xi_{3}=1\right\} \cap\{|\xi|=2\}$ has area $3 \pi$ and touches $|\xi|=1$ in $(0,0,1)$.

Now we are concerned with solutions of the degenerate system

$$
\Delta x_{1}=-\frac{1}{x_{3}}\left(\nabla x_{1} \nabla x_{3}\right), \Delta x_{2}=-\frac{1}{x_{3}}\left(\nabla x_{2} \nabla x_{3}\right), \Delta x_{3}=-\frac{1}{x_{3}}\left(\nabla x_{3} \nabla x_{3}\right)+\frac{1}{2 x_{3}}|\nabla x|^{2}
$$

which turns out to be the system of Euler equation for the integral $E(x)=\int x_{3}$ $|\nabla x(u, v)|^{2} d u d v, x=x(u, v)$. Special interest is given to the variational problem $E(\cdot) \leadsto$ minimum on $\mathfrak{C}(\Gamma, J), J \subset\left\{\xi_{3} \geqq 0\right\}$, since it describes surfaces of least potential energy under gravitational forces, cf. $[1,2]$ for various existence results. Proposition 2 improves the corresponding results 'Theorem 12, 13 in [1]. ${ }^{2}$ )

Proposition 2: Let $J=J_{\varepsilon}=\left\{\xi \in \mathbb{R}^{3}: \xi_{3} \geqq \varepsilon\right\}, \varepsilon>0$, and let $h(\Gamma):=\sup \left\{\xi_{3}\right.$ : $\xi \in I\}$ denote the height of $\Gamma$. Assume that $f \in \mathbb{C}\left(\Gamma, J_{\varepsilon}\right)$ is a solution of $E(\cdot) \rightarrow$ minimum on $\mathbb{E}\left(\Gamma, J_{\epsilon}\right)$, and that either

$$
A(f)=\frac{1}{2} \int_{B}|\nabla f|^{2} d u d v<\frac{\pi R^{2} \varepsilon^{2}}{\dot{\varepsilon}^{2}+\varepsilon R+\frac{1}{2} R^{2}} \quad \text { or } \quad A_{\Gamma, J_{\varepsilon}}<\frac{\varepsilon}{h(\Gamma)} \frac{\pi R^{2} \dot{\varepsilon}^{2}}{\dot{\varepsilon}^{2}+\varepsilon R+\frac{1}{2} R^{2}}
$$

.2) The constants $2^{4} \pi e^{-2} \varepsilon^{2}$ and $(\varepsilon / h) \pi(4 \varepsilon / e)^{2}$ which appear in [1: Theorems 12,13$]$ have to be re: placed by $2^{2} \pi e^{-2} \varepsilon^{2}$ and $(\varepsilon / h) \pi(2 \varepsilon / e)^{2}$, because in Lemma 7 of that article $\mathfrak{\xi}$ denotes 2 -times the mean curvatures which is actually used by these authors. . 
Then $f=f(u, v)$ is contained in the open half space $\left\{\xi_{3}>\varepsilon\right\}$ and furnishes an analytic solution of (2).

We now turn to the proof of Theorem 1 : Let $\chi$ denote the characteristic function of $\Omega^{*}=f^{-1}\left(S_{c}\right)$ and put $\Lambda^{*}(w)=\chi(w) \mathfrak{Q}(f(w))+(1-\chi(w)) H(f(w))$. Following an observation of HILDEBRANDT [11], which was also used in [2], we claim that

$$
D_{U_{\alpha}} f_{*}\left(U_{a}\right)=2 \Lambda^{*} f_{*}\left(U_{1}\right) \times f_{*}\left(U_{2}\right) \quad \text { a.e. on } \Omega \text {. }
$$

In fact, $(3)$ is obvious on $\Omega-\dot{\Omega}^{*}$, while it is a consequence of the conformality relations on $\Omega^{*}$. We refer to $[2,11]$ for explicit calculations. Introduce local coordinates $\varphi: U \rightarrow \mathbb{R}^{3}$, where $\Omega_{1} \subset \Omega$ fulfils $f\left(\Omega_{1}\right) \subset U \subset M$, and let $x(w)=\varphi \circ f(w)$. Then (3) yields

$$
\Delta x^{l}+\Gamma_{i j}^{l}\left\{x_{u}{ }^{i} x_{u}{ }^{j}+x_{v}{ }^{i} x_{v}^{j}\right\}=2 \Lambda^{*}(w) \sqrt{g} g^{l n}\left(x_{u} \wedge x_{v}\right)^{n}
$$

a.e. on $\Omega_{1}$ and for $l=1,2,3$. By virtue of $\left|\Lambda^{*}\right|_{0, \Omega} \leqq \Lambda<\infty$ and arguments from $L_{p}$-theory one immediately infers $f \in H_{p, 10 c}^{2}(\Omega, M) \cap C^{1, \alpha}(\Omega, M)$, for all $p<\infty$ and $\alpha \in(0,1)$. In view of $f \in C^{1}(\Omega, M)$ and $(4)$ we see that $|\Delta x| \leqq$ const $|\nabla x|$ a:e. in $\tilde{\Omega}$ for every $\tilde{\Omega} \subset \subset \Omega$. Hence a technique of HARTMAN and WINTNER is applicable, cf. [ 8 to 10]. In particular one obtains the asymptotic expansions

$$
2 x_{v}(w):=x_{u}-\mathrm{i} x_{v}=(a-\mathrm{i} b)\left(w-w_{0}^{\prime}\right)^{\nu}+o\left(\left|w-w_{0}\right|^{\nu}\right) .
$$

for $w$ close to $w_{0} \in \Omega$. Here the vectors $a, b \in \mathbb{R}^{3}$ fulfil the conformality conditions $\|a\|=\|b\|,\langle a, b\rangle=0$ and $v=v\left(w_{0}\right)$ stands for a non-negative integer. It is now proven as in [2: cf. Lemma 3.11] that (5) in turn implies the density estimate.

$$
\lim _{\ell \rightarrow 0} \sup \frac{1}{\varrho^{2}} \int_{\kappa_{\ell}\left(w_{0}\right)} g_{i j}(x) D_{\mathrm{\alpha}} x^{i} D_{\mathrm{a}} x^{j} d u d v \geqq 2 \pi(v+1)
$$

where $K_{\varrho}\left(w_{0}\right)=\left\{w \in \Omega: d\left(f(w), f\left(w_{0}\right)\right)<\varrho\right\}$. Note that (6) holds for every $w_{0} \in \Omega$, and for some $v \geqq 0$. We are thus in a position to carry over a result of GRÜTER; compare [5: (3.10) Theorem]. ${ }^{3}$ )

Lem ma (cf. [5]): Let $f$ be as above, then the following assertions hold.

a) If $x \leqq 0$ and if $\inf _{\partial \Omega} d\left(f(w), f\left(w_{0}\right)\right) \geqq r$ for some $w_{0} \in \Omega$ where $0<r \leqq \tau$, then

$$
(v+1) 2 \pi r^{2} \leqq D(f)\left\{1+2 \Lambda r+2^{-1}(2 \Lambda r)^{2}\right\} .
$$

b) If $x>0$ and if $\inf _{\partial \Omega} d\left(f(w), f\left(w_{0}\right)\right) \geqq r$ for some $w_{0} \in \Omega$ where $0<r \leqq \min \{\tau$, $\pi / 2 \sqrt{x}\}$, then

$$
\frac{2 \pi(\nu+1)}{x} \leqq D(f)\left\{\frac{1}{\sin ^{2}(r \sqrt{x})}+\frac{2 \Lambda r}{\sin ^{2}(r \sqrt{x})}+\frac{(2 \Lambda)^{2}}{x}\right\} .
$$

Observe that the proof of the theorem in [5:(3.10)] applies to our situation even if $w_{0}$ is a branch point, i.e. $\nabla x\left(w_{0}\right)=0$. In fact in this case $w_{0}$ may not belong to the class of "good" points, compare the definition of the set $A$ in [5]. However, in view

${ }^{8}$ (Note that the left-hand side of (3.1-1) in [5] has to be replaced by $2 \pi / x$ (instead of $2 \pi / \sqrt{x}$ ): 
of what was said before, especially relation (6), it is clear that, in our case, branch points are even "better" points, since $v \geqq 1$ then. This, in turn leads to the estimates of the Lemma, as follows now from a repetition of Grüters argument.

Proceeding with the proof of our theorem, we now assume on the contrary to the assertion that there exists some $w_{0} \in \Omega^{*}$. Since $R=\operatorname{dist}\left(\Gamma, S_{c}\right)$ we obtain inf $\partial \Omega d(f(w)$, $\left.f\left(w_{0}\right)\right) \geqq R \geqq \varrho$. Putting $r: \doteq \varrho$ and $v=0$ in the previous lemma one immediately derives the desired contradiction. We have thus proved that $f(\bar{\Omega}) \subset J_{c}$. The remaining assertions will follow from potential theory

\section{REFERENCES}

[1] Börme, R., Hicdebrandt, S., and F. Tausch: The two-dimensional analogue of the catenary. Pac. J. Math. $88(1980), 247-278$.

[2] Dierkes, U.: Singuläre Variationsprobleme und Hindernisprobleme. Dissertation. Bonn. Math. Schr. 155 (1984), 1-90.

[3] Dierkes, U.: Plateau's problem for surfaces of prescribed mean curvature in given regions. Manuscripta Math. á6 (1986), 313-331.

[4] Gromold, D., Klingenberg, W., und W. Meyer: Riemannsche Geometrie im Großen. Lect. Notes Math. 55 (1968), 1-287.

[5] Grüter, M.: Regularity of weak $H$-surfaces. J. Reine Angew. Math. 329 (1981), 1-15.

[6] GULLIVER; R., and J. SPROCK: Existence theorems for parametric surfaces of prescribed mean curvature. Indiana Univ. Math. J. 22 (1972), 445-472.

[7] Gulirver, R., and J. Sprock: Surfaces of constant mean curvature which have a simple projection. Math. Z. 129 (1972), $95-107$.

[8] Hartman, P., and A. Wintner: On the local behaviour of solutions of non parabolic partial differential equations. Amer. J. Math. 75 (1953), 449-476.

[9] Heinz, E., and S. Hildebrandt: Some remarks on minimal surfaces in Riemannian manifolds. Comm. Pure Appl. Math. 23. (1970), 371-377.

[10] Heinz, E.: Uber das Randverhalten quasilinearer elliptischer Systeme mit isothermen Parametern. Math. Z. 113 (1970), 99-105.

[11] Hundebrandt, S.: Maximum principles for minimal surfaces and for surfaces of continuous mean curvature. Math. Z. 128 (1972), 253-269.

[12] Hildebrand, S.: On the regularity of two-dimensional variational problems with obstructions. Comm. Pure Appl. Math. 25,(1972), 479-496.

[13] Hrdebrandt, S., and.H. KadL: Two-dimensional variational problems with obstruc. tions, and Plateau's problem for $I$-surfaces in a Riemannian manifold. Comm. Pure Appl. Yath. 25 (1972), 187-223.

[14] KaUl, H.: Isoperimetrische Ungleichung und Gauss-Bonnet-Formél für $H$-Flächen in Riemannschen Mannigfaltigkeiten. Arch. Rat. Mech. Analysis 45 (1972), 194-221.

Manuskripteingang: 14.12.1987

VERFASSER :

Dr. Urarich Dierkes

Fachbereich Mathematik der Universität des Saarlandes

D - 6600 Saarbrücken 